\title{
General properties of the Foldy-Wouthuysen transformation and applicability of the corrected original Foldy-Wouthuysen method
}

\author{
Alexander J. Silenkd* \\ Bogoliubov Laboratory of Theoretical Physics, \\ Joint Institute for Nuclear Research, Dubna 141980, Russia, \\ Research Institute for Nuclear Problems, \\ Belarusian State University, Minsk 220030, Belarus
}

\begin{abstract}
General properties of the Foldy-Wouthuysen transformation which is widely used in quantum mechanics and quantum chemistry are considered. Merits and demerits of the original FoldyWouthuysen transformation method are analyzed. While this method does not satisfy the Eriksen condition of the Foldy-Wouthuysen transformation, it can be corrected with the use of the BakerCampbell-Hausdorff formula. We show a possibility of such a correction and propose an appropriate algorithm of calculations. An applicability of the corrected Foldy-Wouthuysen method is restricted by the condition of convergence of a series of relativistic corrections.
\end{abstract}

PACS numbers: 03.65.-w, 11.10.Ef

*alsilenko@mail.ru 


\section{INTRODUCTION}

The Foldy-Wouthuysen (FW) transformation first proposed in the seminal work [1] is now widely used not only in physics but also in quantum chemistry. The FW representation has unique properties. In this representation, the Hamiltonian and all operators are even, i.e., block-diagonal (diagonal in two spinors). Relations between the operators in the FW representation are similar to those between the respective classical quantities. The form of quantum-mechanical operators for relativistic particles in external fields is the same as in the nonrelativistic quantum theory. In particular, the position (Newton-Wigner) operator [2] and the momentum one are equal to $\boldsymbol{r}$ and $\boldsymbol{p}=-i \hbar \nabla$, respectively. The polarization operator for spin-1/2 particles is defined by the Dirac matrix $\boldsymbol{\Pi}$ and is expressed by much more cumbersome formulas in other representations (see [1, 3] ). A great advantage of the FW representation is the simple form of operators corresponding to classical observables. The passage to the classical limit usually reduces to a replacement of the operators in quantum-mechanical Hamiltonians and equations of motion with the corresponding classical quantities. The possibility of such a replacement, explicitly or implicitly used in practically all works devoted to the FW transformation, was rigorously proved in Ref. [4]. Thanks to these properties, the FW representation provides the best possibility of obtaining a meaningful classical limit of relativistic quantum mechanics [1, 5].

There are semi-relativistic and relativistic methods of the FW transformation. We use the term "semi-relativistic" for methods [1, 16 18] using an expansion of a derived blockdiagonal Hamiltonian in even terms of ascending order in 1/c. For the semi-relativistic and the relativistic methods, the zeroth order Hamiltonian is the Schrödinger one and the FW Hamiltonian of a free particle, respectively. The first semi-relativistic method has been proposed by Foldy and Wouthuysen [1]. A FW Hamiltonian obtained by any semi-relativistic method contains a series in powers of the momentum and potential $\left[p /(m c)\right.$ and $\left.V /\left(m c^{2}\right)\right]$, while relativistic methods give a compact relativistic expression for this Hamiltonian for any particle momentum. The first relativistic FW transformation method has been presented in Ref. [19]. Some of the relativistic methods developed in physics are based on unitary transformations [3, 20 22, 29] and a number of these methods uses different approaches [23].

All FW transformation methods applied in quantum chemistry are relativistic. The methods based on unitary transformations follow the approach elaborated by Douglas, Kroll, 
and Hess [24, 25] but often use different transformation operators [6 8, 28]. They allow one to fulfill not only high-order [30] but also arbitrary-order [7, 8, 32, 33] Douglas-Kroll-Hess (DKH) transformations. The DKH transformations expand the FW Hamiltonian in a series in powers of the parameter $V / \sqrt{m^{2} c^{4}+c^{2} \boldsymbol{p}^{2}}$ which is the potential divided by the total kinetic energy. We can also mention the infinite-order two-component method of Barysz and collaborators [35] which is the two-step exact-decoupling approach. Another successful relativistic two-component method is the zeroth-order regular approximation [34]. The exact FW transformation can also be performed in one step [36]. We can refer to the reviews [1113, 15, 26 29] and to the books [9, 10] for more details.

Many transformation methods allowing one to derive a block-diagonal Hamiltonian do not lead to the FW representation (see Refs. [31, 37, 38]). It has been proven in Refs. [31, 37] that the resulting exponential operator of the FW transformation should be odd and Hermitian. Paradoxically, the original FW method [1] explained in Sec. II does not satisfy this requirement and does not lead to the FW representation [37, 39]. Main distinctive features of the FW transformation are considered in Sec. III. To perform the FW transformation, one can use either the exact Eriksen method [37] or one of two different approaches based on successive approximations. These possibilities are analyzed in Sec. IV. The correction of the original FW method is presented in Sec. V] We demonstrate that the corrected original FW method leads to a clear and straightforward calculation of the FW Hamiltonian and can be rather convenient for practical use. To confirm this statement, two examples are given in Sec. VI, For any correct semi-relativistic method, the series of relativistic corrections as a whole may define the exact FW Hamiltonian. However, this takes place only when this series converges. When $p /(m c)>1$ (this situation takes place for an electron near a nucleus), the semi-relativistic methods become inapplicable [7]. Restrictions caused by this circumstance are considered in Sec. VII where the results presented are discussed and summarized.

\section{ORIGINAL FOLDY-WOUTHUYSEN METHOD}

In the general case, a transformation to a new representation described by the wave function $\Psi^{\prime}$ is performed with the unitary operator $U$ :

$$
\Psi^{\prime}=U \Psi=\exp (i S) \Psi
$$


This transformation involves not only the Hamiltonian operator but also the $-i \frac{\partial}{\partial t}$ one. As a result, the Hamiltonian operator in the new representation takes the form

$$
\mathcal{H}^{\prime}=U\left(\mathcal{H}-i \hbar \frac{\partial}{\partial t}\right) U^{-1}+i \hbar \frac{\partial}{\partial t}
$$

or

$$
\mathcal{H}^{\prime}=U \mathcal{H} U^{-1}-i \hbar U \frac{\partial U^{-1}}{\partial t} .
$$

The initial Hamiltonian operator can be split into even and odd operators commuting and anticommuting with the operator $\beta$, respectively:

$$
\mathcal{H}=\beta \mathcal{M}+\mathcal{E}+\mathcal{O}, \quad \beta \mathcal{M}=\mathcal{M} \beta, \quad \beta \mathcal{E}=\mathcal{E} \beta, \quad \beta \mathcal{O}=-\mathcal{O} \beta
$$

The even operators $\mathcal{M}$ and $\mathcal{E}$ and the odd operator $\mathcal{O}$ are diagonal and off-diagonal in two spinors, respectively. This equation is applicable for a particle with any spin if the number of components of a corresponding wave function is equal to $2(2 s+1)$, where $s$ is the spin quantum number. For a Dirac particle, the $\mathcal{M}$ operator usually reduces to the particle rest energy $m c^{2}$ :

$$
\mathcal{H}_{D}=\beta m c^{2}+\mathcal{E}+\mathcal{O}
$$

The Hamiltonian $\mathcal{H}$ is Hermitian for fermions and pseudo-Hermitian (more exactly, $\beta$ pseudo-Hermitian, $\mathcal{H}=\mathcal{H}^{\ddagger} \equiv \beta \mathcal{H}^{\dagger} \beta$ ) for bosons. We assume that the operators $\beta \mathcal{M}, \mathcal{E}$, and $\mathcal{O}$ also possess this property. The transformation operator for bosons is therefore $\beta$ pseudounitary $\left(U^{\dagger}=\beta U^{-1} \beta\right)$. We can mention the existence of bosonic symmetries of the Dirac equation [40].

Equation (2) can be written in the form

$$
\mathcal{H}^{\prime}-i \hbar \frac{\partial}{\partial t}=U\left(\mathcal{H}-i \hbar \frac{\partial}{\partial t}\right) U^{-1}=U\left(\beta \mathcal{M}+\mathcal{E}+\mathcal{O}-i \hbar \frac{\partial}{\partial t}\right) U^{-1} .
$$

This equation allows us to state a rather important property of the FW transformation for a particle in nonstationary (time-dependent) fields. Transformations of two even operators, $\mathcal{E}$ and $-i \hbar \frac{\partial}{\partial t}$, are very similar. As a result, the FW Hamiltonian (except for terms without commutators) contains these operators only in the combination $\mathcal{F}=\mathcal{E}-i \hbar \frac{\partial}{\partial t}$. Therefore, a transition from a stationary to a nonstationary case can be performed with a replacement of $\mathcal{E}$ with $\mathcal{F}$ in all terms containing commutators.

In the present work, we focus our attention on Dirac fermions. 
The original FW method [1] belongs to iteration (step-by-step) methods because a blockdiagonalization of the Hamiltonian is a result of successive iterations. This method allows one to obtain a series of corrections in powers of the momentum and potential to a nonrelativistic Hamiltonian. For the Dirac Hamiltonian written in the form (5), one deduces a series in powers of $\mathcal{O} /\left(m c^{2}\right)$ and $\mathcal{E} /\left(m c^{2}\right)$ including mixed terms. The first step of the $\mathrm{FW}$ transformation is performed with the exponential operator [1]

$$
S=-\frac{i}{2 m c^{2}} \beta \mathcal{O} \text {. }
$$

The transformed Hamiltonian can be written in the form

$$
\begin{gathered}
\mathcal{H}^{\prime}=\mathcal{H}+ \\
-i[S, \mathcal{H}]+\frac{i^{2}}{2 !}[S,[S, \mathcal{H}]]+\frac{i^{3}}{3 !}[S,[S,[S, \mathcal{H}]]]+\ldots \\
-\hbar \dot{S}-\frac{i \hbar}{2 !}[S, \dot{S}]-\frac{i^{2} \hbar}{3 !}[S,[S, \dot{S}]]-\ldots
\end{gathered}
$$

where $[\ldots, \ldots]$ means a commutator. As a result of this transformation, we find

$$
\mathcal{H}^{\prime}=\beta m c^{2}+\mathcal{E}^{\prime}+\mathcal{O}^{\prime}, \quad \beta \mathcal{E}^{\prime}=\mathcal{E}^{\prime} \beta, \quad \beta \mathcal{O}^{\prime}=-\mathcal{O}^{\prime} \beta
$$

The odd operator $\mathcal{O}^{\prime}$ is now $O(1 / m)$. The next step has been made with the operator

$$
S^{\prime}=-\frac{i}{2 m c^{2}} \beta \mathcal{O}^{\prime}
$$

This procedure can be repeated to decrease an order of magnitude of odd terms. It is important that the expression of $\mathcal{H}^{\prime \prime}$ in terms of $\mathcal{E}^{\prime}, \mathcal{O}^{\prime}$ is the same as that of $\mathcal{H}^{\prime}$ in terms of $\mathcal{E}, \mathcal{O}$. This property remains valid at every step.

The original FW method can be simplified if one transforms the operator $\mathcal{F}$ instead of performing separate transformations of the operators $\mathcal{E}$ and $-i \frac{\partial}{\partial t}$.

After the third step, the resulting FW transformation operator is given by

$$
U_{F W}=\exp \left(i S^{\prime \prime}\right) \exp \left(i S^{\prime}\right) \exp (i S)
$$

The initial Dirac Hamiltonian describing a particle in an electromagnetic field is defined by

$$
\mathcal{H}_{D}=\beta m c^{2}+c \boldsymbol{\alpha} \cdot \boldsymbol{\pi}+e \Phi
$$

where $\boldsymbol{\pi}=\boldsymbol{p}-\frac{e}{c} \boldsymbol{A}$ is the kinetic momentum operator, $\Phi$ and $\boldsymbol{A}$ are scalar and vector potentials of the electromagnetic field, respectively, and $e$ is the charge of a particle. For the electron, it is negative $(e=-|e|)$. 
The FW transformation results in [1]

$$
\begin{gathered}
\mathcal{H}_{F W}=\beta\left(m c^{2}+\frac{\boldsymbol{\pi}^{2}}{2 m}-\frac{\boldsymbol{\pi}^{4}}{8 m^{3} c^{2}}\right)+e \Phi-\frac{e \hbar}{2 m c} \boldsymbol{\Pi} \cdot \boldsymbol{B} \\
+\frac{e \hbar}{8 m^{2} c^{2}}(\boldsymbol{\Sigma} \cdot[\boldsymbol{\pi} \times \boldsymbol{E}]-\boldsymbol{\Sigma} \cdot[\boldsymbol{E} \times \boldsymbol{\pi}]-\hbar \nabla \cdot \boldsymbol{E}) .
\end{gathered}
$$

So, the seminal work by Foldy and Wouthuysen has explained spin - magnetic field coupling previously presented by the Pauli equation, the contact (Darwin) interaction proportional to $\nabla \cdot \boldsymbol{E}$, and the "Thomas half" in the terms describing a spin interaction with the electric field.

Evidently, the original FW method is useless when the series of relativistic corrections becomes divergent.

We can emphasize a simple cyclic form of the original FW transformation. All successive iterations are based on the general formulas (7) and (8).

\section{MAIN DISTINCTIVE FEATURES OF THE FOLDY-WOUTHUYSEN TRANS- FORMATION}

The main distinctive features of the FW transformation have been stated in Refs. [31, 37]. The even form of the final Hamiltonian was the only condition of the transformation used by Foldy and Wouthuysen. It can be easily shown that this condition does not define the FW Hamiltonian unambiguously. The result of successive iterations expressed by the equation

$$
U=\ldots \exp \left(i S^{(n)}\right) \ldots \exp \left(i S^{\prime \prime \prime}\right) \exp \left(i S^{\prime \prime}\right) \exp \left(i S^{\prime}\right) \exp (i S)
$$

can be presented in the exponential form:

$$
U=\exp (i \mathfrak{S})
$$

The FW Hamiltonian obtained with this operator is even. We can perform one more unitary transformation with the operator $U^{\prime}=\exp (i \mathfrak{T})$, where the exponential operator $\mathfrak{T}$ is even. This transformation does not add odd terms to the FW Hamiltonian $\mathcal{H}_{F W}$. As a result, the total transformation operator $U=U^{\prime} U_{F W}$ also transforms the initial Hamiltonian to the even form. Since $U^{\prime}$ is an arbitrary even Hermitian and unitary ( $\beta$-pseudo-Hermitian and $\beta$-pseudounitary for bosons) operator, there is an infinity set of transformations leading the initial Hamiltonian to a block-diagonal form. 
The condition eliminating this ambiguity has been proposed by Eriksen [31] and has been substantiated by Eriksen and Kolsrud [37]. The transformation remains unique if the operator $\mathfrak{S}$ in Eq. (15) is odd,

$$
\beta \mathfrak{S}=-\mathfrak{S} \beta
$$

and Hermitian ( $\beta$-pseudo-Hermitian for bosons). Our explanation of the Eriksen method which is given below differs from that presented in original works [31, 37].

Expansion of the exponential operator (15) in series

$$
\exp (i \mathfrak{S})=1+i \mathfrak{S}+\frac{(i \mathfrak{S})^{2}}{2 !}+\frac{(i \mathfrak{S})^{3}}{3 !}+\ldots+\frac{(i \mathfrak{S})^{n}}{n !}+o\left((i \mathfrak{S})^{n}\right)
$$

shows that this condition is equivalent to [31, 37]

$$
\beta U_{F W}=U_{F W}^{\dagger} \beta
$$

We can ascertain that the arbitrary-order DKH transformation [7, 8] satisfies the condition (18).

Thus, the FW transformation operator should satisfy Eq. (18) and should perform the transformation in one step. Eriksen [31] has found an operator possessing these properties. To determine its explicit form, one can introduce the sign operator $\lambda=\mathcal{H} /\left(\mathcal{H}^{2}\right)^{1 / 2}$ and can use the fact that the operator $1+\beta \lambda$ cancels either lower or upper spinor for positive and negative energy states, respectively. It is easy to see that [31]

$$
\lambda^{2}=1, \quad[\beta \lambda, \lambda \beta]=0, \quad[\beta,(\beta \lambda+\lambda \beta)]=0 .
$$

Therefore, the operator of the exact FW transformation has the form

$$
U_{E}=U_{F W}=\frac{1+\beta \lambda}{\sqrt{2+\beta \lambda+\lambda \beta}}, \quad \lambda=\frac{\mathcal{H}}{\left(\mathcal{H}^{2}\right)^{1 / 2}}
$$

The initial Hamiltonian operator, $\mathcal{H}$, is arbitrary. The even operator $\beta \lambda+\lambda \beta$ acting on the wave function with a single nonzero spinor cannot make another spinor be nonzero.

The equivalent form of the operator $U_{E}$ [38] shows that it is properly unitary ( $\beta$ pseudounitary for bosons):

$$
U_{E}=\frac{1+\beta \lambda}{\sqrt{(1+\beta \lambda)^{\dagger}(1+\beta \lambda)}} .
$$

The Eriksen operator (20) can be used for a particle with any spin. In this case, the initial Hamiltonian is given by Eq. (4). 
Evidently, the Eriksen method gives the right relativistic FW Hamiltonian for a free Dirac particle. In this case

$$
\mathcal{E}=0, \quad \lambda=\frac{\beta m c^{2}+\mathcal{O}}{\epsilon}, \quad \mathcal{O}=c \boldsymbol{\alpha} \cdot \boldsymbol{p}, \quad \sqrt{2+\beta \lambda+\lambda \beta}=2\left(1+\frac{m c^{2}}{\epsilon}\right)
$$

with $\epsilon=\sqrt{m^{2} c^{4}+\mathcal{O}^{2}}$. The resulting FW Hamiltonian reads

$$
\mathcal{H}_{F W}=\beta \epsilon
$$

and coincides with the Hamiltonian derived by Foldy and Wouthuysen [1]. A substantiation of the Eriksen method in the more general case of $\mathcal{E} \neq 0$ and $[\mathcal{O}, \mathcal{E}]=0$ has been fulfilled in Ref. [41].

Evidently, the Eriksen operator satisfies Eq. (18). Any additional unitary transformation violates this accordance. For the transformation operator $U=U^{\prime} U_{E}$, where $U^{\prime}=\exp (i \mathfrak{T})$ and the operator $\mathfrak{T}$ is even, block-diagonal Hamiltonians are connected by the even exponential transformation operator.

$$
U^{\dagger} \beta=\beta U_{E}\left(U^{\prime}\right)^{\dagger} \neq \beta U^{\prime} U_{E}
$$

An extra transformation with the odd operator $\mathfrak{T}$ is also inadmissible because its action on the even Hamiltonian $\mathcal{H}_{F W}$ leads to an appearance of odd terms. Therefore, two blockdiagonal Hamiltonians are connected by the even exponential transformation operator.

\section{APPROACHES TO THE FOLDY-WOUTHUYSEN TRANSFORMATION}

The transformation to the FW representation can be carried out by a lot of different methods. The Eriksen method [31] is not iterative and allows one to perform the direct FW transformation. Equations (20) and (21) are convenient to express the FW Hamiltonian as a series of relativistic corrections on powers of $\mathcal{O} / m$ and $\mathcal{E} / m$. One can use the formula

$$
\sqrt{\mathcal{H}^{2}}=\beta m c^{2} \sqrt{1+\frac{\mathcal{H}^{2}-m^{2} c^{4}}{m^{2} c^{4}}}=\beta m c^{2} \sqrt{1+\frac{2 \beta m c^{2} \mathcal{E}+\mathcal{O}^{2}+\mathcal{E}^{2}+\{\mathcal{O}, \mathcal{E}\}}{m^{2} c^{4}}}
$$

and expand $\left(\mathcal{H}^{2}\right)^{-1 / 2}$ in a Taylor series [31, 42]. While needed calculations are cumbersome, they can be made analytically with a computer [42]. We present below the exact FW Hamiltonian calculated by de Vries and Jonker up to terms of the order of $(v / c)^{8}$. They supposed that $\mathcal{O} /\left(m c^{2}\right) \sim(v / c)$ and $\mathcal{E} /\left(m c^{2}\right) \sim(v / c)^{2}$. The result of calculations [42] can 
be presented in a more convenient form [43] via multiple commutators. While the Eriksen method is unapplicable in the nonstationary case, we can use the property formulated below Eq. (6) and rewrite the equation obtained in Ref. [43] as follows:

$$
\begin{aligned}
& \mathcal{H}_{F W}= \beta\left(m c^{2}+\frac{\mathcal{O}^{2}}{2 m c^{2}}-\frac{\mathcal{O}^{4}}{8 m^{3} c^{6}}+\frac{\mathcal{O}^{6}}{16 m^{5} c^{10}}-\frac{5 \mathcal{O}^{8}}{128 m^{7} c^{14}}\right) \\
&+\mathcal{E}- \frac{1}{128 m^{6} c^{12}}\left\{\left(8 m^{4} c^{8}-6 m^{2} c^{4} \mathcal{O}^{2}+5 \mathcal{O}^{4}\right),[\mathcal{O},[\mathcal{O}, \mathcal{F}]]\right\} \\
&+\frac{1}{512 m^{6} c^{12}}\left\{\left(2 m^{2} c^{4}-\mathcal{O}^{2}\right),\left[\mathcal{O}^{2},\left[\mathcal{O}^{2}, \mathcal{F}\right]\right]\right\} \\
&+\frac{1}{16 m^{3} c^{6}} \beta\{\mathcal{O},[[\mathcal{O}, \mathcal{F}], \mathcal{F}]\}-\frac{1}{32 m^{4} c^{8}}[\mathcal{O},[[[\mathcal{O}, \mathcal{F}], \mathcal{F}], \mathcal{F}]] \\
&+ \frac{11}{1024 m^{6} c^{12}}\left[\mathcal{O}^{2},\left[\mathcal{O}^{2},[\mathcal{O},[\mathcal{O}, \mathcal{F}]]\right]\right]+A_{24},
\end{aligned}
$$

where

$$
\begin{aligned}
A_{24}=\frac{1}{256 m^{5} c^{10}} \beta\left(24\left\{\mathcal{O}^{2},([\mathcal{O}, \mathcal{F}])^{2}\right\}-20\left(\left[\mathcal{O}^{2}, \mathcal{F}\right]\right)^{2}-14\left\{\mathcal{O}^{2},\left[\left[\mathcal{O}^{2}, \mathcal{F}\right], \mathcal{F}\right]\right\}\right. \\
-4\left[\mathcal{O},\left[\mathcal{O},\left[\left[\mathcal{O}^{2}, \mathcal{F}\right], \mathcal{F}\right]\right]\right]+\frac{9}{2}\left[\left[\mathcal{O},\left[\mathcal{O},\left[\mathcal{O}^{2}, \mathcal{F}\right]\right]\right], \mathcal{F}\right] \\
\left.-\frac{9}{2}\left[[\mathcal{O},[\mathcal{O}, \mathcal{F}]],\left[\mathcal{O}^{2}, \mathcal{F}\right]\right]+\frac{5}{2}\left[\mathcal{O}^{2},[\mathcal{O},[[\mathcal{O}, \mathcal{F}], \mathcal{F}]]\right]\right) .
\end{aligned}
$$

In $A_{24}$, the first and second subscripts indicate the respective numbers of $\mathcal{F}$ and $\mathcal{O}$ operators in the product. A mistake in the calculation of this term made in Ref. [43] has been corrected in Ref. [21].

Terms of higher orders up to $(v / c)^{12}$ have also been calculated many years ago (see Ref. [42] and references therein). However, the Eriksen method is not practically used in specific calculations. Since the exact equations (19) and (20) contain the square roots of Dirac matrices, they exclude a possibility to obtain a series of relativistic terms with the relativistic FW Hamiltonian of a free particle [1] as the zero-order approximation. This possibility can be realized with the relativistic methods mentioned in Sec. II. Moreover, Eqs. (19) and (20) do not seem to be convenient even for deriving a semi-relativistic FW Hamiltonian. While this possibility has been realized (see Ref. [42] and references therein), a necessity to ensure a commutativity of the numerators and denominators in the expression for $\lambda$ and in Eq. (20) hinders a practical application of the Eriksen method. The FW method 1] and some other semi-relativistic methods are more straightforward. We can note that the use of all semi-relativistic methods in atomic physics and quantum chemistry meet some difficulties caused by their inapplicability at $p /(m c)>1$ [7] (see Sec. VII for more details). 
Nevertheless, the calculation of the FW Hamiltonian by the Eriksen method as a series of relativistic corrections to the zero approximation (Schrödinger Hamiltonian) is very important for checking results obtained by other semi-relativistic and relativistic methods.

The mostly applied approach is characterized by subsequent iterations allowing a determination of both the exponential operator of the FW transformation and the FW Hamiltonian. Intermediate exponential operators obtained at each iteration satisfy the Eriksen condition and are odd and Hermitian. This approach can be realized by many methods [6 10, 17, 18]. Another approach also brings the initial Hamiltonian to a block-diagonal form after subsequent iterations but a resulting exponential transformation operator is not odd. Since all subsequent transformation operators are known, the resulting exponential transformation operator can be corrected by an elimination of even terms in the exponent. The elimination can be made with the use of the Baker-Campbell-Hausdorff (BCH) formula [44]. This formula [44] defines the product of two exponential operators:

$$
\begin{array}{r}
\exp (A) \exp (B)=\exp \left(A+B+\frac{1}{2}[A, B]+\frac{1}{12}[A,[A, B]]-\frac{1}{12}[B,[A, B]]\right. \\
\left.-\frac{1}{24}[A,[B,[A, B]]]+\text { higher order commutators }\right) .
\end{array}
$$

The product of two exponential operators can be calculated with any needed accuracy [45].

When $A=i S, B=i S^{\prime}$ and the operators $S$ and $S^{\prime}$ are odd and Hermitian, the commutators $[A, B]$ and $[A,[B,[A, B]]]$ are even. Therefore, the resulting transformation operator defined by Eq. (14) can be presented in the form $U=\exp (i \mathfrak{R})$ where the operator $\mathfrak{R}$ is not odd and does not satisfy the Eriksen condition (16). Expansion of the operator $U$ in a power series [see Eq. (17)] shows that the equivalent Eriksen condition (18) is also violated.

Thus, the original FW method and other iteration methods do not lead to the FW representation and give only approximate FW Hamiltonians. This fact has been proven by Eriksen and Kolsrud [37] and later by Neznamov [20, 46].

However, there exist possibilities to correct these methods. One can perform one more unitary transformation with the transformation operator $U_{\text {corr }}$ satisfying the relation

$$
U_{c o r r} U=U_{E},
$$

where $U=\exp (i \Re)$ is the resulting transformation operator before the correction and $U_{E}$ is the Eriksen operator (20). The solution of Eq. (27) reduces to an elimination of the even 
part of the operator $\mathfrak{R}$. In the next section, we will consider this problem in more detail with respect to the original FW method.

\section{CORRECTION OF HAMILTONIANS OBTAINED BY THE FOLDY-WOUTHUYSEN METHOD}

In the classical FW method, any subsequent exponential operator is of a smaller order of magnitude than a preceding one. Therefore, the $\mathrm{BCH}$ formula allows one to determine and eliminate an error given by this method. Such a possibility has been first noticed by Eriksen and Kolsrud [37]. If one can neglect $\left[S,\left[S, S^{\prime}\right]\right],\left[S^{\prime},\left[S, S^{\prime}\right]\right]$, and commutators of higher orders as compared with $\left[S, S^{\prime}\right]$, Eq. (26) brings the approximate relation

$$
\exp \left(i S^{\prime}\right) \exp (i S)=\exp \left(\frac{1}{2}\left[S, S^{\prime}\right]\right) \exp \left[i\left(S^{\prime}+S\right)\right]
$$

Since the operator $i\left(S^{\prime}+S\right)$ is odd and the corrected Hamiltonian is even (with a needed accuracy), the left multiplication of the FW transformation operator by the even operator

$$
U_{\text {corr }}=\exp \left(-\frac{1}{2}\left[S, S^{\prime}\right]\right)
$$

does not add any odd terms to the Hamiltonian and allows one to cancel the error of the FW method in the leading order. We can obtain from Eqs. (17) and (10) that the commutator of the two first exponential operators is approximately equal to [39]

$$
\left[S, S^{\prime}\right]=-\frac{\beta}{8 m^{3} c^{6}}\left[\mathcal{O}^{2}, \mathcal{F}\right]
$$

Therefore,

$$
U_{\text {corr }}=\exp \left(\frac{\beta}{16 m^{3} c^{6}}\left[\mathcal{O}^{2}, \mathcal{F}\right]\right) .
$$

This additional transformation eliminates the difference between the results obtained by the original FW method [1] and the Eriksen one [31] in the leading order. For the stationary case, it has been shown in Ref. [37].

In the general case, the corrected transformation operator has the form

$$
U_{E}=U_{c o r r} U \equiv U_{c o r r} \ldots \exp \left(i S^{(n)}\right) \ldots \exp \left(i S^{\prime \prime \prime}\right) \exp \left(i S^{\prime \prime}\right) \exp \left(i S^{\prime}\right) \exp (i S)
$$

and it must be equal to the Eriksen operator. Since the transformation with the operator

$U$ results in the FW Hamiltonian obtained by the original method [1], $\mathcal{H}_{F W}^{(\text {orig) }}$, the corrected 
(right) FW Hamiltonian is given by

$$
\mathcal{H}_{F W}=U_{\text {corr }}\left(\mathcal{H}_{F W}^{(\text {orig })}-i \hbar \frac{\partial}{\partial t}\right) U_{\text {corr }}^{-1}+i \hbar \frac{\partial}{\partial t} .
$$

Since the operators $\mathcal{H}_{F W}$ and $\mathcal{H}_{F W}^{(\text {orig) }}$ are even, the operator $U_{\text {corr }}$ is also even.

\section{EXAMPLES OF THE APPLICATION OF THE CORRECTED ORIGINAL METHOD}

In this section, we will consider two examples of the application of the corrected original FW method and will show the importance of the corrections made.

\section{A. Foldy-Wouthuysen transformation with a calculation of all terms up to the} order of $(v / c)^{6}$

Let us derive the FW Hamiltonian and calculate all terms up to the order of $(v / c)^{6}$ on condition that $\mathcal{E} /\left(m c^{2}\right) \sim(v / c)^{2}, \mathcal{O} /\left(m c^{2}\right) \sim v / c$. The successive steps are given by

$$
\begin{gathered}
S=-\frac{i}{2 m c^{2}} \beta \mathcal{O}, \quad \mathcal{H}^{\prime}=\beta m c^{2}+\mathcal{E}+\beta\left(\frac{\mathcal{O}^{2}}{2 m c^{2}}-\frac{\mathcal{O}^{4}}{8 m^{3} c^{6}}+\frac{\mathcal{O}^{6}}{144 m^{5} c^{10}}\right) \\
-\frac{1}{8 m^{2} c^{4}}[\mathcal{O},[\mathcal{O}, \mathcal{F}]]+\frac{1}{384 m^{4} c^{8}}[\mathcal{O},[\mathcal{O},[\mathcal{O},[\mathcal{O}, \mathcal{F}]]]] \\
+\frac{\beta}{2 m c^{2}}[\mathcal{O}, \mathcal{F}]-\frac{\mathcal{O}^{3}}{3 m^{2} c^{4}}+\frac{\mathcal{O}^{5}}{30 m^{4} c^{8}}-\frac{\beta}{48 m^{3} c^{6}}[\mathcal{O},[\mathcal{O},[\mathcal{O}, \mathcal{F}]]], \\
S^{\prime}=-\frac{i}{4 m^{2} c^{4}}[\mathcal{O}, \mathcal{F}]+i \beta\left(\frac{\mathcal{O}^{3}}{6 m^{3} c^{6}}-\frac{\mathcal{O}^{5}}{60 m^{5} c^{10}}\right)+\frac{i}{96 m^{4} c^{8}}[\mathcal{O},[\mathcal{O},[\mathcal{O}, \mathcal{F}]]], \\
\mathcal{H}^{\prime \prime}=\beta m c^{2}+\mathcal{E}+\beta\left(\frac{\mathcal{O}^{2}}{2 m c^{2}}-\frac{\mathcal{O}^{4}}{8 m^{3} c^{6}}+\frac{\mathcal{O}^{5}}{16 m^{5} c^{10}}\right)-\frac{1}{8 m^{2} c^{4}}[\mathcal{O},[\mathcal{O}, \mathcal{F}]] \\
-\frac{\beta}{8 m^{3} c^{6}}([\mathcal{O}, \mathcal{F}])^{2}+\frac{3}{64 m^{4} c^{8}}\left\{\mathcal{O}^{2},[\mathcal{O},[\mathcal{O}, \mathcal{F}]]\right\}+\frac{5}{128 m^{4} c^{8}}\left[\mathcal{O}^{2},[\mathcal{O}, \mathcal{F}]\right] \\
\quad+\frac{1}{4 m^{2} c^{4}}[[\mathcal{O}, \mathcal{F}], \mathcal{F}]-\frac{\beta}{6 m^{3} c^{6}}\left[\mathcal{O}^{3}, \mathcal{F}\right]-\frac{\beta}{8 m^{3} c^{6}}\left\{\mathcal{O}^{2},[\mathcal{O}, \mathcal{F}]\right\}, \\
S^{\prime \prime}=-\frac{i \beta}{8 m^{3} c^{6}}[[\mathcal{O}, \mathcal{F}], \mathcal{F}]+\frac{i}{12 m^{4} c^{8}}\left[\mathcal{O}^{3}, \mathcal{F}\right]+\frac{i}{16 m^{4} c^{8}}\left\{\mathcal{O}^{2},[\mathcal{O}, \mathcal{F}]\right\} .
\end{gathered}
$$

After the transformation with the operator $S^{\prime \prime}$, the final Hamiltonian obtained by the original FW method takes the form

$$
\begin{gathered}
\mathcal{H}_{F W}^{(\text {orig })}=\beta m c^{2}+\mathcal{E}+\beta\left(\frac{\mathcal{O}^{2}}{2 m c^{2}}-\frac{\mathcal{O}^{4}}{8 m^{3} c^{6}}+\frac{\mathcal{O}^{6}}{16 m^{5} c^{10}}\right)-\frac{1}{8 m^{2} c^{4}}[\mathcal{O},[\mathcal{O}, \mathcal{F}]] \\
-\frac{\beta}{8 m^{3} c^{6}}([\mathcal{O}, \mathcal{F}])^{2}+\frac{3}{64 m^{4} c^{8}}\left\{\mathcal{O}^{2},[\mathcal{O},[\mathcal{O}, \mathcal{F}]]\right\}+\frac{5}{128 m^{4} c^{8}}\left[\mathcal{O}^{2},\left[\mathcal{O}^{2}, \mathcal{F}\right]\right] .
\end{gathered}
$$


It should be corrected according to Eq. (33). In this case, the needed accuracy can be achieved with the single commutator:

$$
\mathcal{H}_{F W}=\mathcal{H}_{F W}^{(\text {orig })}-\left[\frac{1}{2}\left[S, S^{\prime}\right],\left(\mathcal{H}_{F W}^{(\text {orig })}-i \hbar \frac{\partial}{\partial t}\right)\right] .
$$

With the use of Eqs. (34) and (35), we obtain

$$
\mathcal{H}_{F W}=\mathcal{H}_{F W}^{(\text {orig })}+\left[\frac{\beta}{16 m^{3} c^{6}}\left[\mathcal{O}^{2}, \mathcal{F}\right],\left(\mathcal{F}+\beta \frac{\mathcal{O}^{2}}{2 m c^{2}}\right)\right] .
$$

As a result,

$$
\begin{gathered}
\mathcal{H}_{F W}=\beta m c^{2}+\mathcal{E}+\beta\left(\frac{\mathcal{O}^{2}}{2 m c^{2}}-\frac{\mathcal{O}^{4}}{8 m^{3} c^{6}}+\frac{\mathcal{O}^{6}}{16 m^{5} c^{10}}\right)-\frac{1}{8 m^{2} c^{4}}[\mathcal{O},[\mathcal{O}, \mathcal{F}]] \\
+\frac{\beta}{16 m^{3} c^{6}}\{\mathcal{O},[[\mathcal{O}, \mathcal{F}], \mathcal{F}]\}+\frac{3}{64 m^{4} c^{8}}\left\{\mathcal{O}^{2},[\mathcal{O},[\mathcal{O}, \mathcal{F}]]\right\}+\frac{1}{128 m^{4} c^{8}}\left[\mathcal{O}^{2},\left[\mathcal{O}^{2}, \mathcal{F}\right]\right] .
\end{gathered}
$$

This equation agrees with the result obtained by the Eriksen method and expressed by Eq. (24).

\section{B. Foldy-Wouthuysen transformation with a calculation of all terms up to the} order of $m^{-4}$

It is also instructive to calculate all terms up to the order of $m^{-4}$ on the condition that $\mathcal{E} \sim \mathcal{O}$. In this case, the successive exponential transformation operators are given by

$$
\begin{gathered}
S=-\frac{i}{2 m c^{2}} \beta \mathcal{O}, \\
S^{\prime}=-\frac{i}{4 m^{2} c^{4}}[\mathcal{O}, \mathcal{F}]+\frac{i}{6 m^{3} c^{6}} \beta \mathcal{O}^{3}+\frac{i}{96 m^{4} c^{8}}[\mathcal{O},[\mathcal{O},[\mathcal{O}, \mathcal{F}]]] \\
S^{\prime \prime}=-\frac{i \beta}{8 m^{3} c^{6}}[[\mathcal{O}, \mathcal{F}], \mathcal{F}]+\frac{i}{12 m^{4} c^{8}}\left[\mathcal{O}^{3}, \mathcal{F}\right]+\frac{i}{16 m^{4} c^{8}}\left\{\mathcal{O}^{2},[\mathcal{O}, \mathcal{F}]\right\}, \\
S^{\prime \prime \prime}=-\frac{i}{16 m^{4} c^{8}}[[[\mathcal{O}, \mathcal{F}], \mathcal{F}], \mathcal{F}] .
\end{gathered}
$$

The Hamiltonian has the form

$$
\begin{aligned}
& \mathcal{H}_{F W}^{(\text {orig })}=\beta m c^{2}+\mathcal{E}+\beta\left(\frac{\mathcal{O}^{2}}{2 m c^{2}}-\frac{\mathcal{O}^{4}}{8 m^{3} c^{6}}\right)-\frac{1}{8 m^{2} c^{4}}[\mathcal{O},[\mathcal{O}, \mathcal{F}]]-\frac{\beta}{8 m^{3} c^{6}}([\mathcal{O}, \mathcal{F}])^{2} \\
& +\frac{3}{64 m^{4} c^{8}}\left\{\mathcal{O}^{2},[\mathcal{O},[\mathcal{O}, \mathcal{F}]]\right\}+\frac{5}{128 m^{4} c^{8}}\left[\mathcal{O}^{2},\left[\mathcal{O}^{2}, \mathcal{F}\right]\right]+\frac{1}{32 m^{4} c^{8}}[[\mathcal{O}, \mathcal{F}],[[\mathcal{O}, \mathcal{F}], \mathcal{F}]] .
\end{aligned}
$$

To calculate all terms up to the order of $m^{-4}$, one needs take into account commutators with the exponential transformation operators $S^{\prime}$ and $S^{\prime \prime}$ :

$$
\mathcal{H}_{F W}=\mathcal{H}_{F W}^{(\text {orig })}-\left[\frac{1}{2}\left[S,\left(S^{\prime}+S^{\prime \prime}\right)\right],\left(\mathcal{H}_{F W}^{(\text {orig })}-i \hbar \frac{\partial}{\partial t}\right)\right] .
$$


The implication of the operator $S^{\prime \prime}$ into the correction procedure distinguishes this example from the precedent one.

Equations (39)- (41) result in

$$
\begin{gathered}
\mathcal{H}_{F W}=\mathcal{H}_{F W}^{(\text {orig })}+\left[\frac{\beta}{16 m^{3} c^{6}}\left[\mathcal{O}^{2}, \mathcal{F}\right],\left(\mathcal{F}+\beta \frac{\mathcal{O}^{2}}{2 m c^{2}}\right)\right] \\
-\frac{1}{32 m^{4} c^{8}}[[\mathcal{O},[[\mathcal{O}, \mathcal{F}], \mathcal{F}]], \mathcal{F}] .
\end{gathered}
$$

Since $[[\mathcal{O}, \mathcal{F}],[[\mathcal{O}, \mathcal{F}], \mathcal{F}]]-[[\mathcal{O},[[\mathcal{O}, \mathcal{F}], \mathcal{F}]], \mathcal{F}]=-[\mathcal{O},[[[\mathcal{O}, \mathcal{F}], \mathcal{F}], \mathcal{F}]]$, the corrected FW Hamiltonian has the form

$$
\begin{gathered}
\mathcal{H}_{F W}=\beta m c^{2}+\mathcal{E}+\beta\left(\frac{\mathcal{O}^{2}}{2 m c^{2}}-\frac{\mathcal{O}^{4}}{8 m^{3} c^{6}}\right)-\frac{1}{8 m^{2} c^{4}}[\mathcal{O},[\mathcal{O}, \mathcal{F}]] \\
+\frac{\beta}{16 m^{3} c^{6}}\{\mathcal{O},[[\mathcal{O}, \mathcal{F}], \mathcal{F}]\}+\frac{3}{64 m^{4} c^{8}}\left\{\mathcal{O}^{2},[\mathcal{O},[\mathcal{O}, \mathcal{F}]]\right\} \\
+\frac{1}{128 m^{4} c^{8}}\left[\mathcal{O}^{2},\left[\mathcal{O}^{2}, \mathcal{F}\right]\right]-\frac{1}{32 m^{4} c^{8}}[\mathcal{O},[[[\mathcal{O}, \mathcal{F}], \mathcal{F}], \mathcal{F}]]
\end{gathered}
$$

This expression also agrees with Eq. (24).

We can conclude that the corrected original FW method ensures a straightforward derivation of the FW Hamiltonian.

\section{DISCUSSION AND SUMMARY}

The wonderful achievements of Eriksen are the formulation and the substantiation of conditions of transformation to the FW representation, the derivation of the exact FW transformation operator, the proof of an approximateness of the original FW method, and the discovery of the possibility of its correction. The Eriksen method gives the correct FW Hamiltonian for a free particle and also in the more general case [41] of $\mathcal{E} \neq 0$ and $[\mathcal{O}, \mathcal{E}]=0$. In the general nonrelativistic case, the Eriksen formula (20) allows one to present the FW Hamiltonian as a series of relativistic corrections to the Schrödinger Hamiltonian (see Ref. [42]). However, this series (as well as a series given by any nonrelativistic method) is divergent when $p /(m c)>1$.

It has been shown in Ref. [7] that the use of all semi-relativistic methods in quantum chemistry is restricted due to their divergence at $p /(m c)>1$. Evidently, this takes place in a small region near a nucleus. In the classical theory, the energy of the electron is given by

$$
E=\sqrt{m^{2} c^{4}+c^{2} \boldsymbol{p}^{2}}-\frac{Z e^{2}}{r}
$$


where $Z$ is the atomic number. The small region of the series divergence is defined by the approximate condition

$$
r \lesssim Z r_{0}
$$

where $r_{0}=e^{2} /\left(m c^{2}\right)=2.818 \times 10^{-13} \mathrm{~cm}$ is the classical electron radius. In this small region, FW wave eigenfunctions are undefined. When this is not admissible, one should use appropriate relativistic methods. For example, the method developed in Ref. [7] gives a convergent series because the expansion parameter contains the kinetic energy in the denominator and is always less than 1 .

Inside of the region of the series convergence, semi-relativistic and relativistic methods of the FW transformation should give equivalent results. However, an existence of the series divergence restricts an application of all semi-relativistic methods in quantum chemistry. The corrected FW method is perfect in all cases when the series divergence does not appear (for example, for a description of a particle in a trap). The derivation of the Hamiltonian $\mathcal{H}_{F W}^{(\text {orig) }}$ reproduced in Sec. II represents a straightforward computer cycle based on the general formulas (77) and (86). The next computer cycle is the calculation of the resulting exponential transformation operator with the BCH formula (26). The product of two exponential operators defined by this formula can be obtained with any needed accuracy. Then, one needs to find the operator $U_{\text {corr }}$ satisfying the relation (27) and eliminating the even part of the exponential operator $\mathfrak{R}$. The FW Hamiltonian can by finally obtained by the transformation of the operator $\mathcal{H}_{F W}^{(\text {orig) }}$ with the operator $U_{\text {corr }}$. This transformation is given by Eq. (33). An applicability of the corrected FW method is demonstrated by the two examples presented in Sec. VI.

We can conclude that the correction of iterative methods with the $\mathrm{BCH}$ formula allows one to use these methods for a derivation of the FW Hamiltonians with a needed accuracy but their applicability is restricted by the condition of the series convergence.

Let us also consider the electron density at the position $\boldsymbol{r}_{A}$ of a specific nucleus $A$. In Refs. [10, 14], the corresponding Dirac operator has been found to be

$$
\begin{gathered}
\widehat{O}=\sum_{i}^{N} \widehat{O}\left(\boldsymbol{r}_{i}\right) \quad \text { with } \quad \widehat{O}\left(\boldsymbol{r}_{i}\right)=\delta^{(3)}\left(\boldsymbol{r}_{i}-\boldsymbol{r}_{A}\right) \\
=\delta\left(x_{i}-x_{A}\right) \delta\left(y_{i}-y_{A}\right) \delta\left(z_{i}-z_{A}\right) .
\end{gathered}
$$

In the FW representation, this operator takes the form $U_{F W} \widehat{O} U_{F W}^{\dagger}$. The expectation value 
for the electron density then reads $[10,14$

$$
\rho_{i i}(\boldsymbol{r})=\left\langle U_{F W} \psi_{i}\left|U_{F W} \widehat{O} U_{F W}^{\dagger}\right| U_{F W} \psi_{i}\right\rangle .
$$

This formula has been used for specific calculations [14]. The charge distribution obtained with the FW transformation can significantly differ from the corresponding nonrelativistic charge distribution.

\section{ACKNOWLEDGEMENTS}

This work was supported in part by the Belarusian Republican Foundation for Fundamental Research (Grant No. \$14D-007) and by the Heisenberg-Landau Program of the German Ministry for Science and Technology (Bundesministerium für Bildung und Forschung).

[1] L. L. Foldy, S. A. Wouthuysen, Phys. Rev. 78, 29 (1950).

[2] T. D. Newton, E. P. Wigner, Rev. Mod. Phys. 21, 400 (1949).

[3] A. J. Silenko, J. Math. Phys. 44, 2952 (2003).

[4] A. J. Silenko, Pis'ma Zh. Fiz. Elem. Chast. Atom. Yadra 10, 144 (2013) [Phys. Part. Nucl. Lett. 10, 91 (2013)].

[5] J. P. Costella, B. H. J. McKellar, Am. J. Phys. 63, 1119 (1995).

[6] D. Kędziera and M. Barysz, Chem. Phys. Lett. 446, 176 (2007); F. Aquilante et al, J. Comput. Chem. 31, 224 (2010); D. Peng, N. Middendorf, F. Weigend, and M. Reiher, J. Chem. Phys. 138, 184105 (2013).

[7] M. Reiher and A. Wolf, J. Chem. Phys. 121, 2037 (2004).

[8] M. Reiher and A. Wolf, J. Chem. Phys. 121, 10945 (2004); A. Wolf and M. Reiher, J. Chem. Phys. 124, 064102 (2006); 124, 064103 (2006).

[9] K. G. Dyall and K. Faegri, Introduction to relativistic quantum chemistry (Oxford University Press, Oxford, 2007).

[10] M. Reiher and A. Wolf, Relativistic Quantum Chemistry: The Fundamental Theory of Molecular Science (Wiley-VCH, Weinheim, 2009).

[11] M. Reiher, Sequential decoupling of negative-energy states in DouglasKrollHess theory. In: Handbook of Relativistic Quantum Chemistry, ed. by W. Liu ((Springer-Verlag, Berlin, 2015). 
[12] J. Autschbach, Coord. Chem. Rev. 251, 1796 (2007).

[13] T. Nakajima, K. Hirao, Chem. Rev. 112, 385 (2012).

[14] R. Mastalerz, R. Lindh, M. Reiher, Chem. Phys. Lett. 465, 157 (2008).

[15] M. Reiher, WIREs Comput. Mol. Sci. 2, 139 (2012).

[16] S. Stephani, Ann. Phys. (Leipzig) 470, 12 (1965).

[17] F. A. Reuse, Electrodynamique et Optique Quantiques (Presses Polytechniques et Universitaires Romandes, Lausanne, 2007).

[18] Y. Hinschberger and P.-A. Hervieux, Phys. Lett. A 376, 813 (2012).

[19] G. Morpurgo, Nuovo Cimento 15, 624 (1960).

[20] V. P. Neznamov, Fiz. Elem. Chastits At. Yadra 37, 152 (2006) [Phys. Part. Nucl. 37, 86 (2006)].

[21] A. J. Silenko, Phys. Rev. A 77, 012116 (2008).

[22] A. J. Silenko, Phys. Rev. A 91, 022103 (2015).

[23] E. I. Blount, Phys. Rev. 128, 2454 (1962); A. J. Silenko, Theor. Math. Phys. 105, 1224 (1995); 112, 922 (1997); K. Y. Bliokh, Europhys. Lett. 72, 7 (2005); Phys. Lett. A 351, 123 (2006); P. Gosselin, A. Berard, and H. Mohrbach, Eur. Phys. J. B 58, 137 (2007); Phys. Lett. A 368, 356 (2007); P. Gosselin, J. Hanssen, and H. Mohrbach, Phys. Rev. D 77, 085008 (2008); P. Gosselin and H. Mohrbach, Eur. Phys. J. C 64, 495 (2009).

[24] M. Douglas and N. M. Kroll, Ann. Phys. 82, 89 (1974).

[25] B. A. Hess, Phys. Rev. A 32, 756 (1985); 33, 3742 (1986).

[26] M. Reiher, Theor. Chem. Acc. 116, 241 (2006).

[27] W. Liu, Mol. Phys. 108, 1679 (2010).

[28] D. Peng and M. Reiher, J. Chem. Phys. 136, 244108 (2012).

[29] D. Peng and M. Reiher, Theor. Chem. Acc. 131, 1081 (2012).

[30] T. Nakajima and K. Hirao, Chem. Phys. Lett. 329, 511 (2000); J. Chem. Phys. 113, 7786 (2000); A. Wolf, M. Reiher, and B. A. Hess, J. Chem. Phys. 117, 9215 (2002); C. van Wllen, J. Chem. Phys. 120, 7307 (2004).

[31] E. Eriksen, Phys. Rev. 111, 1011 (1958).

[32] M. Reiher and A. Wolf, Phys. Lett. A 360, 603 (2007).

[33] D. Peng and K. Hirao, J. Chem. Phys. 130, 044102 (2009). 
[34] C. Chang, M. Pelissier, and P. Durand, Phys. Scr. 34, 394 (1986); E. van Lenthe, E. J. Baerends, and J. G. Snijders, J. Chem. Phys. 99, 4597 (1993); 101, 9783 (1994).

[35] M. Barysz, A. J. Sadlej, J. G. Snijders, Int. J. Quantum Chem. 65, 225 (1997); M. Barysz, J. Chem. Phys. 114, 9315 (2001); M. Barysz, A. J. Sadlej, J. Chem. Phys. 116, 2696 (2002); D. Kędziera and M. Barysz, Chem. Phys. Lett. 393, 521 (2004).

[36] K. G. Dyall, J. Chem. Phys. 106, 9618 (1997); 109, 4201 (1998); 115, 9136 (2001); J. Comput. Chem. 23, 786 (2002); K. G. Dyall and T. Enevoldsen, J. Chem. Phys. 111, 10000 (1999); M. Filatov and D. Cremer, J. Chem. Phys. 119, 11526 (2003); 122, 064104 (2005); W. Kutzelnigg and W. Liu, J. Chem. Phys. 123, 241102 (2005); Mol. Phys. 104, 2225 (2006); W. Liu and D. Peng, J. Chem. Phys. 125, 044102 (2006); M. Filatov and K. G. Dyall, Theor. Chem. Acc. 117, 333 (2007); W. Liu and W. Kutzelnigg, J. Chem. Phys. 126, 114107 (2007); M. Ilias̆ and T. Saue, J. Chem. Phys. 126, 064102 (2007); D. Peng, W. Liu, Y. Xiao, and L. Cheng, J. Chem. Phys. 127, 104106 (2007); W. Liu and D. Peng, J. Chem. Phys. 131, 031104 (2009); J. Sikkema, L. Visscher, T. Saue, and M. Ilias̆, J. Chem. Phys. 131, 124116 (2009).

[37] E. Eriksen and M. Korlsrud, Nuovo Cimento Suppl. 18, 1 (1960).

[38] V.P. Neznamov and A. J. Silenko, J. Math. Phys. 50, 122302 (2009).

[39] E. de Vries, Fortschr. Phys. 18, 149 (1970).

[40] V. M. Simulik, I. Yu. Krivsky, Bosonic symmetries of the massless Dirac equation Adv. Appl. Clifford Alg. 8, 69 (1998); Phys. Lett. A 375, 2479 (2011); V. M. Simulik, I. Yu. Krivsky, I. L. Lamer, Ukr. J. Phys. 58, 523 (2013); TWMS Journ. Appl. Engin. Math. 3, 46 (2013).

[41] A. J. Silenko, Pis'ma Zh. Fiz. Elem. Chast. Atom. Yadra 10, 321 (2013) [Phys. Part. Nucl. Lett. 10, 198 (2013)].

[42] E. de Vries, J. E. Jonker, Nucl. Phys. B 6, 213 (1968).

[43] A. J. Silenko, Teor. Mat. Fiz. 176, 189 (2013) [Theor. Math. Phys. 176, 987 (2013)].

[44] H. Baker, Proc. Lond. Math. Soc. (1) 34, 347 (1902); (1) 35, 333 (1903); (Ser. 2) 3, 24 (1905); J. Campbell, Proc. Lond. Math. Soc. 28, 381 (1897); 29, 14 (1898); F. Hausdorff, Ber. Verh. Saechs. Akad. Wiss. Leipzig, Math.-Phys. Kl. 58, 19 (1906). This formula has also been obtained by H. Poincaré, Compt. Rend. Acad. Sci. Paris 128, 1065 (1899); Camb. Philos. Trans. 18, 220 (1899).

[45] E. B. Dynkin, Dokl. Akad. Nauk SSSR 57, 323 (1947) (in Russian). 
[46] V.P. Neznamov, Voprosy Atomnoj Nauki i Tekhniki, Seriya Teoreticheskaya i Prikladnaya Fizika (in Russian) 2, 21 (1988). 